Pré-Publicações do Departamento de Matemática

Universidade de Coimbra

Preprint Number 06-37

\title{
ON SOME SPECIAL CLASSES OF CONTINUOUS MAPS
}

\author{
MARIA MANUEL CLEMENTINO AND DIRK HOFMANN
}

ABSTRACT: We present a survey on recent study of special continuous maps, like biquotient, triquotient, proper, perfect, open and étale maps and a selection of open problems in this area.

\section{Special morphisms of Top}

Triquotient maps were introduced by E. Michael in [24] as those continuous maps $f: X \rightarrow Y$ for which there exists a map (_) $)^{\sharp}: \mathcal{O} X \rightarrow \mathcal{O} Y$ such that, for every $U, V$ in the lattice $\mathcal{O} X$ of open subsets of $X$ :

(T1) $U^{\sharp} \subseteq f(U)$,

(T2) $X^{\sharp}=Y$,

(T3) $U \subseteq V \Rightarrow U^{\sharp} \subseteq V^{\sharp}$,

(T4) $\left(\forall y \in U^{\sharp}\right)\left(\forall \Sigma \subseteq \mathcal{O} X\right.$ directed) $f^{-1}(y) \cap U \subseteq \bigcup \Sigma \Rightarrow \exists S \in \Sigma: y \in S^{\sharp}$.

It is easy to check that, if $f: X \rightarrow Y$ is an open surjection, then the direct image $f\left({ }_{-}\right): \mathcal{O} X \rightarrow \mathcal{O} Y$ satisfies (T1)-(T4). If $f: X \rightarrow Y$ is a retraction, so that there exists a continuous map $s: Y \rightarrow X$ with $f \circ s=1_{Y}$, then (_) $)^{\sharp}:=$ $s^{-1}\left(_{-}\right)$satisfies (T1)-(T4). Moreover, if $f: X \rightarrow Y$ is a proper surjection (by proper map we mean a closed map with compact fibres: see [2]), then $U^{\sharp}:=Y \backslash f(X \backslash U)$ fulfills (T1)-(T4). That is, open surjections, retractions and proper surjections are triquotient maps. But there are triquotient maps which are neither of these maps (cf. [3, 15] for examples). However, we do not know whether these three classes of maps describe completely triquotient maps, in the sense we state now:

Received August 24, 2005.

The authors acknowledge partial financial assistance by Centro de Matemática da Universidade de Coimbra/FCT and by Unidade de Investigação e Desenvolvimento Matemática e Aplicações da Universidade de Aveiro/FCT.

This text will be part of the book "Open Problems in Topology 2", to be published by Elsevier BV. 
Question 1. Is it true that any triquotient map can be factored through (possibly infinitely many) open surjections, proper surjections and retractions?

T. Plewe in [26] related triquotient maps to Topological Grothendieck Descent Theory (see [17]). We recall that a continuous map $f: X \rightarrow Y$ is an effective descent map if its pullback functor $f^{*}: \operatorname{Top} / Y \rightarrow \operatorname{Top} / X$, that assigns to each $g: W \rightarrow Y$ its pullback along $f$, is monadic. If $f^{*}$ is premonadic, then $f$ is a descent map (see $[18,19]$ ). Descent maps are exactly universal quotient maps [10], or pullback-stable quotient maps, that is quotient maps whose pullback along any map is still a quotient. We point out here that this class of maps was introduced independently by B. Day and M. Kelly [10], by E. Michael [23], under the name biquotient maps, and by O. Hájek [11], as limit lifting maps. Effective descent maps turned out to be very difficult to describe topologically. The only characterisation that is known is due to J. Reiterman and W. Tholen [27] and uses heavily ultrafilter convergence. (We will concentrate on ultrafilter convergence later in this work.)

Problem 2. Find a characterisation of topological effective descent maps in terms of the topologies or the Kuratowski closures.

One possible approach to this problem could be via the existence of a map between the topologies that resembles the map (_) $)^{\sharp}$ introduced by E. Michael to define triquotient maps. Indeed, it is interesting to notice that one can characterise several of these classes of morphisms using a map $\mathcal{O} X \rightarrow \mathcal{O} Y$ as follows: a continuous map $f: X \rightarrow Y$ is:

- a universal quotient map (= biquotient $=$ limit lifting $=$ descent $)$ if and only if there exists a map (_) $)^{\sharp} \mathcal{O} X \rightarrow \mathcal{O} Y$ satisfying (T1)-(T3) and

(U4) $\forall y \in Y \forall \Sigma \subseteq \mathcal{O} X$ directed $f^{-1}(y) \subseteq \bigcup \Sigma \Rightarrow \exists S \in \Sigma: y \in S^{\sharp}$.

- a proper surjection if and only if there exists a map (_) $)^{\sharp}: \mathcal{O} X \rightarrow \mathcal{O} Y$ satisfying (T1)-(T3) and, for every $U \in \mathcal{O} X$

(P4) $\left(\forall y \in U^{\sharp}\right)(\forall \Sigma \subseteq \mathcal{O} X$ directed $) f^{-1}(y) \cap U \subseteq \bigcup \Sigma \Rightarrow \exists S \in \Sigma: y \in$ $S^{\sharp}$ and $f^{-1}(y) \subseteq S$. 
- an open surjection if and only if there exists a map (_) $)^{\sharp}: \mathcal{O} X \rightarrow \mathcal{O} Y$ satisfying (T1)-(T3) and, for every $U \in \mathcal{O} X$

(O4) $(\forall y \in f(U))\left(\forall \Sigma \subseteq \mathcal{O} X\right.$ directed) $f^{-1}(y) \cap U \subseteq \bigcup \Sigma \Rightarrow \exists S \in \Sigma: y \in S^{\sharp}$.

Problem 3. Describe effective descent maps via the existence of a map $\left(_{-}\right)^{\sharp}$ similar to those described above.

A possible approach to Problem 3 might be making use of the following result, that we could prove only for maps between finite topological spaces. It is based on the existence of a map between the lattices of locally closed subsets (i.e. the subsets which are an intersection of an open and a closed subset), which we will denote by $\mathcal{L} C\left(_{-}\right)$.

Theorem. If $X$ and $Y$ are finite spaces, a continuous map $f: X \rightarrow Y$ is effective descent if and only if, for every pullback $g: W \rightarrow Z$ of $f$, there exists a map $\left(_{-}\right)^{\sharp}: \mathcal{L} C(W) \rightarrow \mathcal{L} C(Z)$ such that, for every $A, B \in \mathcal{L} C(W)$,

(1) $A^{\sharp} \subseteq g(A)$;

(2) $W^{\sharp}=Z$;

(3) $A \subseteq B \Rightarrow A^{\sharp} \subseteq B^{\sharp}$;

(4) $\forall z \in Z g^{-1}(z) \subseteq A \Rightarrow z \in A^{\sharp}$.

We believe that the work of G. Richter [28] may be inspiring to attack Problem 3.

In the pioneer work [15], G. Janelidze and M. Sobral describe several classes of maps using convergence, whenever $X$ and $Y$ are finite spaces.

Theorem. [15] For finite spaces $X, Y$, a continuous map $f: X \rightarrow Y$ is:

(a) a universal quotient if and only if, for every $y_{0}, y_{1} \in Y$ with $y_{1} \rightarrow y_{0}$, there exist $x_{0}, x_{1} \in X$ such that $x_{1} \rightarrow x_{0}, f\left(x_{0}\right)=y_{0}$ and $f\left(x_{1}\right)=y_{1}$;

(b) an effective descent map if and only if, for every $y_{0}, y_{1}, y_{2} \in Y$ with $y_{2} \rightarrow y_{1} \rightarrow y_{0}$, there exist $x_{0}, x_{1}, x_{2} \in X$ such that $x_{2} \rightarrow x_{1} \rightarrow x_{0}$ and $f\left(x_{i}\right)=y_{i}$, for $i=0,1,2$;

(c) a triquotient map if and only if, for every $y_{0}, \cdots, y_{n} \in Y$ with $y_{n} \rightarrow \cdots \rightarrow y_{0}$, there exist $x_{0}, \cdots, x_{n} \in X$ such that $x_{n} \rightarrow \cdots \rightarrow x_{0}$ and $f\left(x_{i}\right)=y_{i}$, for $i=0, \cdots, n$;

(d) a proper map if and only if, for every $x_{1} \in X$ and $y_{0} \in Y$ with $f\left(x_{1}\right) \rightarrow y_{0}$, there exists $x_{0} \in X$ such that $x_{1} \rightarrow x_{0}$ and $f\left(x_{0}\right)=y_{0}$; 
(e) an open map if and only if, for every $x_{0} \in X$ and $y_{1} \in Y$ with $y_{1} \rightarrow f\left(x_{0}\right)$, there exists $x_{1} \in X$ such that $x_{1} \rightarrow x_{0}$ and $f\left(x_{1}\right)=y_{1}$;

(f) a perfect map if and only if, for every $x_{1} \in X$ and $y_{0} \in Y$ with $f\left(x_{1}\right) \rightarrow y_{0}$, there exists a unique $x_{0} \in X$ such that $x_{1} \rightarrow x_{0}$ and $f\left(x_{0}\right)=y_{0}$

(g) a local homeomorphism (or étale map) if and only if, for every $x_{0} \in X$ and $y_{1} \in Y$ with $y_{1} \rightarrow f\left(x_{0}\right)$, there exists a unique $x_{1} \in X$ such that $x_{1} \rightarrow x_{0}$ and $f\left(x_{1}\right)=y_{1}$.

We recall that a continuous map $f: X \rightarrow Y$ is perfect if it is proper and Hausdorff (i.e., if $f(x)=f\left(x^{\prime}\right)$ and $x \neq x^{\prime}$, there exists $U, V \in \mathcal{O} X$ with $x \in U, x^{\prime} \in V$ and $U \cap V=\emptyset$ ), and that it is a local homeomorphism - or an étale map - if it is open and, for each $x \in X$, there exists $U \in \mathcal{O} X$ such that $x \in U$ and $f_{\mid U}: U \rightarrow f(U)$ is a homeomorphism.

This work led us to investigate the extension of these characterisations to maps between (infinite) topological spaces. The right setting to use convergence turned out to be the ultrafilter convergence. As a side result we also obtained, together with W. Tholen, a useful characterisation of exponentiable maps via convergence (see [9]) we will mention in Section 2.

The results corresponding to (a), (d), (e), (f) were either known or easy to obtain; in fact, the characterisation of universal quotient maps using convergence is the basis for the definition of limit lifting maps by Hájek, and the descriptions of open, proper and perfect maps are straightforward (see [4]). Statement (b) corresponds, in the infinite case, to the ReitermanTholen characterisation of effective descent maps. Indeed, although this is not completely evident in the original formulation [27], the notions and techniques introduced in [4] clarify completely the analogy between these characterisations. In the latter paper, we also generalized the JanelidzeSobral-Clementino characterisation of triquotient maps (c) to the infinite case, as we will explain later.

After that, only a characterisation of local homeomorphisms, using ultrafilter convergence, remained unknown to us. To explain the problem we first state the characterisation of proper, perfect and open maps. 
Proposition. (1) A continuous map $f: X \rightarrow Y$ is proper (perfect) if and only if, for each ultrafilter $\mathfrak{x}$ in $X$ with $f[\mathfrak{x}] \rightarrow y$ in $Y$, there exists a (unique) $x$ in $X$ such that $\mathfrak{x} \rightarrow x$ and $f(x)=y$ :

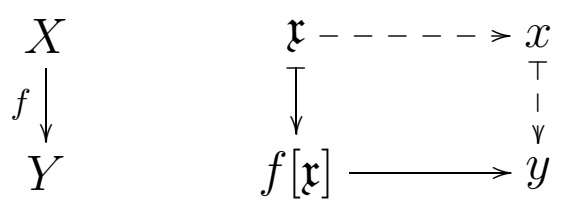

(2) A continuous map $f: X \rightarrow Y$ is open if and only if, for each $x \in X$ and each ultrafilter $\mathfrak{y}$ in $Y$ with $\mathfrak{y} \rightarrow f(x)$ in $Y$, there exists an ultrafilter $\mathfrak{x}$ in $X$ such that $\mathfrak{x} \rightarrow x$ and $f(\mathfrak{x})=\mathfrak{y}$ :
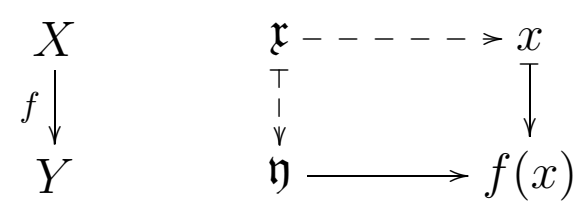

Analysing the characterisations of proper and perfect map, we conjectured that, from the characterisation of open map, one could obtain a characterisation of local homeomorphism imposing the unicity of the lifting of the convergence $\mathfrak{y} \rightarrow f(x)$.

Indeed, the parallelism between the two situations becomes evident once we observe that, if we denote by $\delta_{f}: X \rightarrow X \times_{Y} X, x \mapsto(x, x)$, the continuous map induced by the pullback property of the (pullback) diagram below

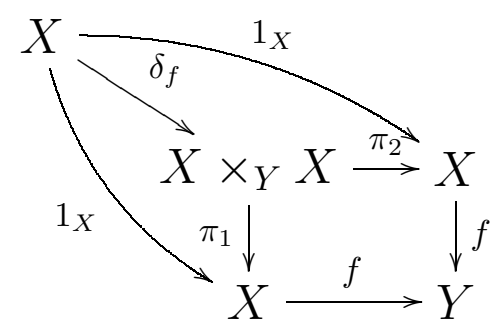

then:

- a continuous map $f: X \rightarrow Y$ is perfect if and only if $f$ and $\delta_{f}$ are proper maps,

- a continuous map $f: X \rightarrow Y$ is a local homeomorphism if and only if $f$ and $\delta_{f}$ are open maps. 
Eventually we have shown, together with G. Janelidze [8], that our conjecture was wrong. Calling a continuous map $f: X \rightarrow Y$ having the unicity of the lifting of $\mathfrak{y} \rightarrow f(x)$ described above a discrete fibration (using the parallelism with categorical discrete fibrations), one has:

Proposition. [8] Every local homeomorphism is a discrete fibration (and the converse is false).

We could prove that the two notions coincide under some conditions on the domain of the map. For that, given a cardinal number $\lambda$, we call a topological space $X$ a $\lambda$-space if the character of $X$ is at most $\lambda$ and each subset of $X$ with cardinality less than $\lambda$ is closed.

Theorem. [8] If $X$ is a $\lambda$-space, for some cardinal $\lambda$, then, for continuous maps with domain $X$, local homeomorphisms and discrete fibrations coincide.

Among $\lambda$-spaces one has the indiscrete spaces $(=0$-spaces $)$, the Alexandrov spaces $(=1$-spaces $)$ and the first countable $T_{1}$-spaces $\left(=\aleph_{0}\right.$-spaces $)$.

Problem 4. Characterise those topological spaces $X$ such that, for a continuous map $f: X \rightarrow Y, f$ is a local homeomorphism if and only if it is a discrete fibration.

In order to formulate more results and problems in this context we need to consider iterations of the ultrafilter convergence. The right way of doing this is making it a functorial process. There are two natural choices in this direction. We may use the ultrafilter functor $U: \mathbf{R e l} \rightarrow \operatorname{Rel}$, which assigns to each set $X$ its set of ultrafilters $U X$, and to each relation $r$ : $X \nrightarrow Y$ the corresponding relation $U r: U X \nrightarrow U Y$ (see for instance [1]). For maps $f: X \rightarrow Y, U f: U X \rightarrow U Y$ is the usual map; for simplicity we write $U f(\mathfrak{x})=f[\mathfrak{x}]$. The most valuable functor in this study is the functor Conv : Top $\rightarrow$ URS, where URS is the category of ultrarelational spaces and convergence preserving maps (see [4]), which assigns to each topological space $X$ the space $\operatorname{Conv}(X)$; here $\operatorname{Conv}(X)$ is the set consisting of pairs $(\mathfrak{x}, x)$, where $x$ is a point and $\mathfrak{x}$ is an ultrafilter converging to $x$ in $X$, equipped with a convergence structure as follows: first we consider the 
map $p: \operatorname{Conv}(X) \rightarrow X$ with $p(\mathfrak{x}, x)=x$; an ultrafilter $\mathfrak{X}$ converges to $(\mathfrak{x}, x)$ in $\operatorname{Conv}(X)$ if $p[\mathfrak{X}]=\mathfrak{x}$. Each continuous map $f: X \rightarrow Y$ induces a map $\operatorname{Conv}(f): \operatorname{Conv}(X) \rightarrow \operatorname{Conv}(Y)$ with $(\mathfrak{x}, x) \mapsto(f[\mathfrak{x}], f(x))$, which preserves the convergence structure (see [4] for details).

It is clear that we can consider instead Conv : URS $\rightarrow$ URS. Furthermore, the map $p: \operatorname{Conv}(X) \rightarrow X$ preserves the structure, so that it defines a natural transformation $p:$ Conv $\rightarrow 1_{\text {URS }}$.

This functor Conv is an excellent tool to describe our classes of maps via their lifting of convergence. For that we need to consider (possibly transfinite) iterations of the functor Conv : URS $\rightarrow$ URS, as described in [4].

For an ordinal number $\alpha$ we call a continuous map $f: X \rightarrow Y$ between ultrarelational (or topological) spaces $\alpha$-surjective if, for every $\beta<\alpha$, $\operatorname{Conv}^{\beta}(f): \operatorname{Conv}^{\beta}(X) \rightarrow \operatorname{Conv}^{\beta}(Y)$ is surjective; $f: X \rightarrow Y$ is $\Omega$-surjective if $\operatorname{Conv}^{\alpha}(f)$ is surjective for every ordinal $\alpha$.

Theorem. [4] For a continuous map $f: X \rightarrow Y$ between topological spaces,

(1) $f$ is 1-surjective if and only if it is surjective;

(2) $f$ is 2-surjective if and only if it is a universal quotient map (if and only if it is a descent map);

(3) $f$ is 3-surjective if and only if it is an effective descent map;

$(\Omega) f$ is $\Omega$-surjective if and only if it is a triquotient map.

Similarly to Problem 2, we may formulate the following

Problem 5. Study the properties of the classes of 4-surjective, ..., nsurjective, $\omega$-surjective maps, and possible characterisations of these classes using the topologies (or even the sequential closures).

Concerning assertion $(\Omega)$ above, it is shown in [4] that, for a continuous map $f: X \rightarrow Y, f$ is a $\Omega$-surjection if and only if $f$ is a $\lambda_{Y}$-surjection, where $\lambda_{Y}$ is the successor of the cardinal of $Y$. This covers the result already known for a continuous map between finite spaces: $f$ is a triquotient (hence $\Omega$-surjective) if and only if it is $\omega$-surjective (see $[3,15]$ ).

Problem 6. Characterise those topological spaces $Y$ such that, for a continuous map $f: X \rightarrow Y, f$ is $\Omega$-surjective if and only if it is $\omega$-surjective. 
Using the functor $U: \operatorname{Rel} \rightarrow$ Rel instead of Conv $:$ URS $\rightarrow$ URS, one can also iterate $U$ and formulate the notions of $U$ - $\alpha$-surjective map, for any continuous map $f: X \rightarrow Y$ between topological spaces. (We will keep the name $\alpha$-surjective for Conv- $\alpha$-surjective maps.)

It is easy to check that every 3 -surjective map is $U$-3-surjective, i.e. every effective descent map is $U$-3-surjective.

Question 7. Is every U-3-surjective map effective descent?

Question 8. If the answer to the previous question is negative, is the class of effective descent maps the least pullback-stable class containing the U-3-surjective maps?

Furthermore, the functor Conv may be also useful to characterise local homeomorphisms as special discrete fibrations. For instance, one may ask the following:

Question 9. Is every continuous map $f$ such that both $f$ and $\operatorname{Conv}(f)$ are discrete fibrations a local homeomorphism?

There is another problem in Topological Descent Theory, described in the sequel, that justifies the study of local homeomorphisms, or étale maps, using convergence.

The notion of an effective (global-)descent map can be generalised by considering instead of all morphisms with codomain $Y$ a well-behaved subclass $\mathrm{E}(Y)$ of morphisms. One important example is obtained by taking $\mathrm{E}$ the class of all étale maps, so that $\mathrm{E}(Y)$ is the category of étale bundles over the space $Y$. A continuous map $f: X \rightarrow Y$ is called effective étale-descent if the pullback functor $f^{*}: \mathrm{E}(Y) \rightarrow \mathrm{E}(X)$ is monadic. For finite spaces $X$ and $Y$, the problem of characterising effective étale-descent maps was solved by G. Janelidze and M. Sobral:

Theorem. [16] The morphism $f: X \rightarrow Y$ in FinTop is effective étaledescent if and only if the functor $\varphi: \mathrm{Z}(\mathrm{Eq}(f)) \rightarrow Y$ is an equivalence of categories.

Here $\operatorname{Eq}(f)$ is the equivalence relation on $X$ induced by $f$, and $Z(\operatorname{Eq}(f))$ is the category having as objects the points of $X$; a morphism from $x$ to $x^{\prime}$ 
is an equivalence class of zigzags in $X$, that is,

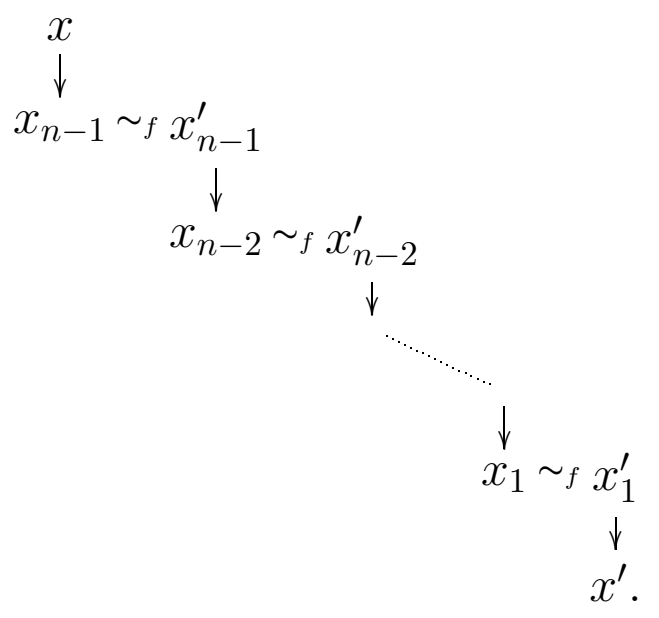

Now $\varphi: \mathrm{Z}(\operatorname{Eq}(f)) \rightarrow Y$ is an equivalence of categories if and only if

(1) $f: X \rightarrow f(X)$ is a quotient map,

(2) $\mathrm{Z}(\mathrm{Eq}(f))$ is a preorder and

(3) $f: X \rightarrow Y$ is essentially surjective on objects (i.e. for every $y \in Y$ there exists $x \in X$ such that $f(x) \rightarrow y \rightarrow f(x))$.

The obvious question is now how to transport this result into the context of all topological spaces.

Question 10. Characterise effective étale-descent maps $f: X \rightarrow Y$ between arbitrary topological spaces.

A possible solution to the problem above requires most likely translations of point-convergence notions and arguments to (ultra)filter-convergence ones. Both notions, of local homeomorphism and quotient map, should be considered in this problem via ultrafilter convergence. We have already mentioned the study of local homeomorphisms using convergence developed in [8]; possible descriptions of quotients, and their relations to zigzags, are studied in [13].

\section{Corresponding morphisms in related categories}

We study now the same problems in categories related to Top. Here same will be used with two meanings: either we consider characteristic categorical properties of the morphisms, or we deal with topological categories whose 
objects and morphisms have a description similar to the (ultrafilter) convergence description of Top. (We will not focus in this latter subject since it would take us too far from our purpose here. But we refer the reader to [5].)

We start by considering some important supercategories (improvements) of Top. The interest in these categories has its roots in the fact that many categorically defined constructions either cannot be carried out in Top or destroy properties of spaces or maps. In order to perform these constructions, topologists move (temporarily) outside Top into larger but better behaved environments such as the category PsTop of pseudotopological spaces and continuous (i.e. convergence preserving) maps. Recall that a pseudotopology on a set $X$ may be described as a convergence relation $\mathfrak{x} \rightarrow x$ between ultrafilters $\mathfrak{x}$ on $X$ and points $x \in X$ such that the principal ultrafilter $\dot{x}$ converges to $x$. The category PsTop contains Top as a full and reflective subcategory; in fact, it is the quasitopos hull of Top (see [12] for details). Being in particular a quasitopos, PsTop is locally cartesian closed and therefore the class of effective descent morphisms coincides with the class of quotient maps.

A pseudotopology on a set $X$ is called a pretopology if it is closed under intersections in the sense that $\bigcap_{\mathfrak{y} \rightarrow x} \mathfrak{y} \subseteq \mathfrak{x}$ implies $\mathfrak{x} \rightarrow x$, for each ultrafilter $\mathfrak{x} \in U X$ and each $x \in X$. Hence convergence to a point $x$ is completely determined by the neighbourhood filter $\bigcap_{\mathfrak{y} \rightarrow x} \mathfrak{y}$. Together with continuous maps pretopological spaces form the category PrTop. In [12] is is shown that PrTop is the extensional topological hull of Top, that is, the smallest extensional topological category containing Top 'nicely'. However, in contrary to PsTop, the category PrTop is not cartesian closed. Exponentiable pretopological spaces are characterised in [22] as those spaces where each point has a smallest neighbourhood. The map version of this result is established in [29]: it states that a continuous map $f: X \rightarrow Y$ between pretopological spaces is exponentiable if and only if each $x \in X$ has a neighbourhood $V$ such that, for each ultrafilter $\mathfrak{x}$ in $X$, if $V \in \mathfrak{x}$ and $f[\mathfrak{x}] \rightarrow f(x)$ in $Y$, then $\mathfrak{x} \rightarrow x$ in $X$. Whereas exponentiable objects and morphisms are fully understood in PrTop, effective descent maps have not been described yet. 
Question 11. Characterise effective descent maps $f: X \rightarrow Y$ between pretopological spaces.

The study of these classes of maps is also interesting in metric-like structures. Together with metric spaces we also consider premetric spaces. By a premetric space we mean a set $X$ together with a map $a: X \times X \rightarrow[0, \infty]$ such that $a(x, x)=0$ and $a(x, z) \leq a(x, y)+a(y, z)$, for any $x, y, z \in X$; that is, a premetric is a, possibly infinite, reflexive and not necessarily symmetric distance. We consider now the categories Met, of metric spaces and nonexpansive maps, and PMet, of premetric spaces and non-expansive maps.

Exponentiable and effective descent maps between metric - and more generally premetric - spaces are characterised in [7] and [6] respectively. We list here the results which might serve, together with the corresponding results for topological spaces, as a guideline for the study of these classes of maps in approach spaces as outlined below.

Theorem. A non-expansive map $f:(X, a) \rightarrow(Y, b)$ between premetric spaces is exponentiable in $\mathbf{P M e t}$ if and only if, for each $x_{0}, x_{2} \in X, y_{1} \in Y$ and $u_{0}, u_{1} \in \mathbb{R}$ such that $u_{0} \geq b\left(f\left(x_{0}\right), y_{1}\right), u_{1} \geq b\left(y_{1}, f\left(x_{2}\right)\right)$ and

$$
\begin{aligned}
u_{0}+u_{1} & =\max \left\{a\left(x_{0}, x_{2}\right), b\left(f\left(x_{0}\right), y_{1}\right)+b\left(y_{1}, f\left(x_{2}\right)\right)\right\}<\infty, \\
\forall \varepsilon & >0 \exists x_{1} \in f^{-1}\left(y_{1}\right): a\left(x_{0}, x_{1}\right)<u_{0}+\varepsilon \text { and } a\left(x_{1}, x_{2}\right)<u_{1}+\varepsilon .
\end{aligned}
$$

Theorem. A non-expansive map $f:(X, a) \rightarrow(Y, b)$ between metric spaces is exponentiable in Met if and only if it is exponentiable in PMet and has bounded fibres.

Theorem. A morphism $f:(X, a) \rightarrow(Y, b)$ in PMet (Met) is effective descent if and only if

$$
\forall y_{0}, y_{1}, y_{2} \in Y \quad b\left(y_{2}, y_{1}\right)+b\left(y_{1}, y_{0}\right)=\inf _{x_{i} \in f^{-1}\left(y_{i}\right)} a\left(x_{2}, x_{1}\right)+a\left(x_{1}, x_{0}\right) .
$$

Approach spaces were introduced by R. Lowen [20] as a natural generalisation of both topological and metric spaces. They can be defined in many different ways; however, the most convenient presentation for our purpose uses ultrafilter convergence (see also [5]): an approach space $(X, a)$ is a pair consisting of a set $X$ and a "numerified" convergence structure 
$a: U X \times X \rightarrow[0, \infty]$ such that

$$
0 \geq a(\dot{x}, x) \quad \text { and } \quad U a(\mathfrak{X}, \mathfrak{x})+a(\mathfrak{x}, x) \geq a\left(m_{X}(\mathfrak{X}), x\right) .
$$

A map $f: X \rightarrow Y$ between approach spaces $(X, a)$ and $(Y, b)$ is called non-expansive if $a(\mathfrak{x}, x) \geq b(U f(\mathfrak{x}), f(x))$, for all $\mathfrak{x} \in U X$ and $x \in X$. We denote by App the category of approach spaces and non-expansive maps. So far, in App little is known about exponentiable objects and morphisms and nothing about effective descent morphisms, though one may conjecture that a combination of the known results in Top and Met will provide characterisations of these classes of objects and maps in App.

Question 12. Characterise exponentiable objects and maps in App.

Question 13. Characterise effective descent maps in App.

Exponentiable objects in approach theory are studied in [21, 14], and the following sufficient condition is obtained.

Theorem. [14] An approach space $(X, a)$ is exponentiable provided that, for each $\mathfrak{X} \in U^{2} X, x \in X$ with a $\left(m_{X}(\mathfrak{X}), x\right)<\infty$, each $u_{0}, u_{1} \in[0, \infty)$ with $u_{1}+u_{0}=a\left(m_{X}(\mathfrak{X}), x\right)$, and each $\varepsilon>0$, there exists an ultrafilter $\mathfrak{x}$ such that

$$
U a(\mathfrak{X}, \mathfrak{x}) \leq u_{1}+\varepsilon \quad \text { and } \quad a(\mathfrak{x}, x) \leq u_{0}+\varepsilon .
$$

We conjecture that the condition above is also necessary for $(X, a)$ to be exponentiable. A first step towards a solution to Question 13 is to define the functor Conv in the context of approach theory. This, by the way, would also open the door to carry the notion of triquotient map to App.

2.1. Unif. We turn now our attention to the category Unif of uniform spaces and uniformly continuous maps. The question regarding exponentiable maps was settled by $\mathrm{S}$. Niefield [25]. In the result below $(X, \mathcal{U})$ and $(Y, \mathcal{V})$ are uniform spaces, with $\mathcal{U}$ and $\mathcal{V}$ the sets of entourages of the uniformities. For $f: X \rightarrow Y, A \subseteq X \times X$ and $y, y^{\prime} \in Y$, let $A_{y, y^{\prime}}:=A \cap\left(f^{-1}(y) \times f^{-1}\left(y^{\prime}\right)\right)$.

Theorem. A morphism $f: X \rightarrow Y$ in Unif is exponentiable if and only if there exists $U_{0} \in \mathcal{U}$ satisfying 
(1) for all $U \in \mathcal{U}, V_{U}=\left\{\left(y, y^{\prime}\right) \in Y \times Y \mid U_{0 y, y^{\prime}}=U_{y y^{\prime}}\right\} \in \mathcal{V}$,

(2) there exists $V_{0} \in \mathcal{V}$ such that the projection $\pi_{1}: U_{0 y y^{\prime}} \rightarrow f^{-1}(y)$ is a surjection whenever $\left(y, y^{\prime}\right) \in V_{0}$.

In particular we have that a uniform space is exponentiable if and only its uniformity has a smallest entourage. However, nothing is known about effective descent maps in Unif.

Question 14. Characterise effective descent maps $f: X \rightarrow Y$ in Unif.

\section{References}

[1] M. Barr, Relational algebras, in: Lecture Notes in Math. 137 (Springer, Berlin 1970), pp. 39-55.

[2] N. Bourbaki, Topologie Générale, (Hermann, Paris 1961).

[3] M. M. Clementino, On finite triquotient maps, J. Pure Appl. Algebra 168 (2002) 387-389.

[4] M. M. Clementino and D. Hofmann, Triquotient maps via ultrafilter convergence, Proc. Amer. Math. Soc. 130 (2002) 3423-3431.

[5] M.M. Clementino and D. Hofmann, Topological features of lax algebras, Appl. Categ. Structures 11 (2003) 267-286.

[6] M.M. Clementino and D. Hofmann, Effective descent morphisms in categories of lax algebras, Appl. Categ. Structures 12 (2004) no. 5-6, 413-425.

[7] M.M. Clementino and D. Hofmann, Exponentiation in $\mathbb{V}$-categories, Top. Appl., to appear.

[8] M. M. Clementino, Dirk Hofmann and George Janelidze, Local homeomorphisms via ultrafilter convergence, Proc. Amer. Math. Soc. 133 (2005) 917-922.

[9] M. M. Clementino, Dirk Hofmann and Walter Tholen, The convergence approach to exponentiable maps, Port. Math. 60 (2003) 139-160.

[10] B. J. Day and G. M. Kelly, On topological quotient maps preserved by pullbacks or products, Proc. Cambridge Philos. Soc. 67 (1970) 553-558.

[11] O. Hájek, Notes on quotient maps, Comment. Math. Univ. Carolinae 7 (1966) 319-323.

[12] H. Herrlich, E. Lowen-Colebunders and F. Schwarz, Improving Top: PrTop and PsTop, in: Category Theory at Work (Heldermann Verlag, Berlin 1991), 21-34.

[13] D. Hofmann, An algebraic description of regular epimorphisms in topology, J. Pure Appl. Algebra 199 (2005) 71-86.

[14] D. Hofmann, Exponentiation for unitary structures, Top. Appl. (to appear).

[15] G. Janelidze and M. Sobral, Finite preorders and topological descent I, J. Pure Appl. Algebra 175 (2002) 187-205.

[16] G. Janelidze and M. Sobral, Finite preorders and topological descent II: étale descent, $J$. Pure Appl. Algebra 174 (2002) 303-309.

[17] G. Janelidze, M. Sobral and W. Tholen, Beyond Barr Exactness: Effective Descent Morphisms, in: Categorical Foundations. Special Topics in Order, Topology, Algebra, and Sheaf Theory, Encyclopedia of Mathematics and its Applications 97, Cambridge University Press (2004), pp. 359-405. 
[18] G. Janelidze and W. Tholen, How algebraic is the change-of-base functor?, in: Proceedings Conference Category Theory, Como 1990, Lecture Notes in Mathematics 1488 (Springer, Berlin 1991), pp. 174-186.

[19] G. Janelidze and W. Tholen, Facets of descent I, Applied Cat. Struct. 2 (1994) 245-281.

[20] R. Lowen, Approach Spaces: The missing link in the Topology-Uniformity-Metric Triad, Oxford Mathematical Monographs (Oxford University Press, Oxford 1997).

[21] R. Lowen and M. Sioen, On the multitude of monoidal closed structures on UAP, Top. Appl. 137 (2004) 215-223.

[22] E. Lowen and G. Sonck, Exponentiable objects and cartesian closedness in the construct PrTop, Appl. Categ. Structures 1 (1993) 345-360.

[23] E. Michael, Bi-quotient maps and cartesian products of quotient maps, Ann. Inst. Fourier, Grenoble 18 (1968) 287-302.

[24] E. Michael, Complete spaces and tri-quotient maps, Illinois J. of Math. 21 (1977) 716-733.

[25] S. B. Niefield, Cartesianness: topological spaces, uniform spaces, and affine schemes, J. Pure Appl. Algebra 23 (1982) 147-167.

[26] T. Plewe, Localic triquotient maps are effective descent morphisms, Math. Proc. Cambridge Philos. Soc. 122 (1997) 17-43.

[27] J. Reiterman and W. Tholen, Effective descent maps of topological spaces, Top. Appl. 57 (1994) 53-69.

[28] G. Richter, Exponentiable maps and triquotients in Top, J. Pure Appl. Algebra 168 (2002) 99-105.

[29] G. Richter, A characterization of exponentiable maps in PrTop, Appl. Categ. Structures 11 (2003) 261-265.

Maria Manuel Clementino

Departamento de Matemática, Univ. de Coimbra, 3001-454 Coimbra, Portugal

E-mail address: mmc@mat.uc.pt

$U R L:$ http://www.mat.uc.pt/ mmc

DiRK HOFMANN

Departamento de Matemática, Universidade de Aveiro, 3810-193 Aveiro, Portugal

E-mail address: dirk@mat.ua.pt 